\title{
Femtosecond optical absorption studies of nonequilibrium electronic processes in high $T_{G}$ superconductors
}

\author{
J. M. Chwalek, C. Uher, ${ }^{\text {a) }}$ J. F. Whitaker, and G. A. Mourou \\ Ultrafast Science Laboratory, University of Michigan, Ann Arbor, Michigan 48109 \\ J. Agostinelli and M. Lelental \\ Corporate Research Laboratories, Eastman Kodak Company, Rochester, New York 14650
}

(Received 10 May 1990; accepted for publication 17 August 1990)

\begin{abstract}
We report the results of femtosecond optical transient absorption experiments performed on the superconducting compounds $\mathrm{YBa}_{2} \mathrm{Cu}_{3} \mathrm{O}_{7} \ldots x(x \sim 0)$ and $\mathrm{Bi}_{2} \mathrm{Sr}_{2} \mathrm{Ca}_{2} \mathrm{Cu}_{3} \mathrm{O}_{30}+\delta(\delta \sim 0)$ and nonsuperconducting $\mathrm{YBa}_{2} \mathrm{Cu}_{3} \mathrm{O}_{6+y}(y<0.4)$ for sample temperatures ranging from

$\sim 7 \mathrm{~K}$ to room temperature. Nonequilibrium heating was found to occur on a subpicosecond time scale. A distinct, dramatic increase in the relaxation time was observed for the superconducting samples as the sample temperature was lowered below the critical temperatures of the respective films. Accompanying the increase in relaxation time was an increase in the peak fractional transmissivity change. No such changes were observed for the nonsuperconducting YBCO sample. We believe the above described behavior is electronic in origin and intimately related to the saperconductivity of the compounds.
\end{abstract}

The ability to generate and time resolve nonequilibrium electron distributions using short laser pulses (laser pulse durations less than or comparable to electronphonon energy relaxation time) has produced important information regarding the thermal properties of the carriers and phonons in metals. ${ }^{\hat{3}}$ Short duration laser pulses ( $\sim 6$ ps) have also been used to study the electron-phonon relaxation rate in single-crystal, nonsuperconducting lanthanum cuprate. ${ }^{4}$ Recently, femtosecond pump-probe neasurements of the room-temperature optical properties of several high $T_{c}$ superconducting and nonsuperconducting compounds were reported. ${ }^{5}$ In this letter, we present evidence of nonequilibrium carrier heating on a subpicosecond time scale in thin-fim samples of the superconducting compounds $\mathrm{YBa}_{2} \mathrm{Cu}_{3} \mathrm{O}_{7-x}(x \sim 0)$ and $\mathrm{Bi}_{2} \mathrm{Sr}_{2} \mathrm{Ca}_{2} \mathrm{Cu}_{3} \mathrm{O}_{10+\delta}(\delta \sim 0)$ and nonsuperconducting $\mathrm{YBa}_{2} \mathrm{Cu}_{3} \mathrm{O}_{6+y} \quad(y<0.4)$. In particular, we focus on the relaxation time of the hot carrier distribution as a function of sample temperature both above and below the critical temperature.

The optical properties of superconducting cuprates are generally quite complicated and not well understood. This was particularly true for early studies, which were performed at a time when techniques for producing good samples were not widely available. Recently, thorough studies of the optical properties in the visible wavelength range (the range we are most concerned about for this study) have become available. In particular, the broad absorption feature centered near $2.5 \mathrm{eV}$ common to the copperoxide perovskite materials is believed to be due to an interband transition. 6,7 If the optically probed states are near the Fermi level a thermomodulation process can occur.

A number of effects can contribute to the transient response in thermomodulation experiments, including effects due to lattice expansion, Fermi level shifting, smearing of the electron occupancy near the Fermi levei, and

a) Dept. of Physics, University of Michigan. electron-phonon interaction. ${ }^{1,3}$ With the presence of an interband electronic ransition promoting electrons to states lying near the Fermi level (such as the $d$-band to $p$-band iransition that occurs in noble metals), it has been established that the dominant contribution to the transient response is Ferni smearing resulting from electron heating. 1,3

Our experimental setup is similar to transient thermomodulation pump-probe experiments reported in Ref. 2 . In our case the short pulse light source was a coliding-pulse mode-locked dye laser that produced pulses of $\sim 100 \mathrm{fs}$ duration at a $100 \mathrm{MHz}$ repetition rate and a wavelength of $630 \mathrm{~nm}(1.98 \mathrm{eV})$. The pump beam provided ultrafast heating which in turn produced a change in the refractive index. This was directly measured with a second beam (polarized perpendicularly to the pump beam) by refiecting the probe beam of the surface of the film, or as in our case, by passing it directly through the semitransparent film in a transient absorption mode. The resultant change in transmissivity was sensed by a photodiode and recorded versus the pump-probe delay time. The pump beam intensity was kept less than $6 \mathrm{~mW}$ while the probe intensity was less than $1.5 \mathrm{~mW}$. The resulting relatively low fuences of $\sim 10 \mathrm{~kJ} / \mathrm{cm}^{2}$ minimized the bulk heating of the film (estimated to be less than $10 \mathrm{~K})^{8}$ while still maintaining a good signal-to-noise ratio.

The $c$-axis oriented $\mathrm{Bi}_{2} \mathrm{Sr}_{2} \mathrm{Ca}_{2} \mathrm{Cl}_{3} \mathrm{O}_{10}$ film used for our study was produced using a metal organic decomposition (MOD) technique on a $\mathrm{MgO}$ substrate. ${ }^{9}$ The film contained crystallites ranging in size from 10 to $50 \mu \mathrm{m}$. With the high spatial resolution afiorded by the laser we were able to single out an individual crystallite. ac susceptibility measurements indicated an onset to diamagnetism at 108 $\mathrm{K}$ and a broad transition commonly associated with weak intergrain coupling as well as intergrowths of the $T_{c}=85 \mathrm{~K}$ phase present in the Bi-Sr-Ca-Cu-O system.

Shown in Fig. I is the normalized transmissivity change versus pump-probe delay time obtained for the 


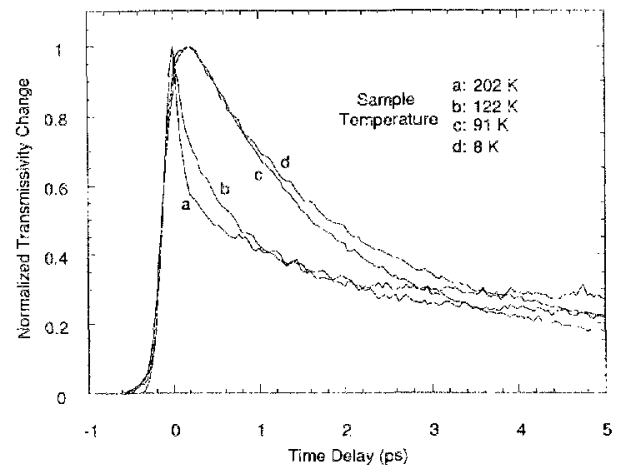

FIG. 1. Transmissivity change normalized to unity is plotted relative to the pump-probe delay time for the $\mathrm{Bi}_{2} \mathrm{Sr}_{2} \mathrm{Ca}_{2} \mathrm{Cu}_{3} \mathrm{O}_{10}$ fim at a few sample temperatures. The 0 ps delay time was chosen to coincide with the maximum transmissivity change of the $202 \mathrm{~K}$ trace.

$\mathrm{Bi}_{2} \mathrm{Sr}_{2} \mathrm{Ca}_{2} \mathrm{Cu}_{3} \mathrm{O}_{10}$ film at several sample temperatures. The essential features of the data which provide evidence for a nonequilibrim carrier effect include a rapid transient near 0 ps which follows the cross correlation of the pump and probe pulses and a consequent relaxation which occurs on a subpicosecond kime scale. In measured $d$-band metals this type of signature can be interpreted as an initial heating of the conduction electrons which thermalize rapidly via electron-electron scattering. This hot-electron distribution then rapidly thermalizes with the lattice by energy transfer through electron-phonon scattering. For later delay times heat diffusion out of the Huminated volume of the film will occur. Because of the extremely poor thermal conductivity of the high $T_{c}$ cuprates, this diffusion process would be extremely slow ( $>1$ ns). The most interesting features of the data include the dependence of the relaxation time on sample temperature. A distinct, dramatic increase in the relaxation lime occurs for sample temperatures below the critical temperature of the film. Another interesting feature for sample temperatures below $T_{c}$ is a delay in the rising edge near 0 ps. This behavior is not understood at this point.

The dependence of the relaxation time with sample temperature for the BSCCO film is displayed graphically in Fig. 2(a). The relaxation time was found by a least-squares fit to the declining part of the data using a decaying exponential plus a constant term. The exponential term models the relaxation of the hot carriers while the constant term simpiy models the excess heat which escapes by the much slower process of heat diffusion. As can be seen from the figure, the relaxation time actually increases slightly with increasing temperature as $T_{c}$ is approached before dropping. An abrupt change in relaxation time over such a small sample temperature range would not be expected in a conventional metal since the electron-phonon coupling is only very weakly temperature dependent.

Figure $2(b)$ plots the peak fractional transmissivity change, $(\Delta T / T)_{\max }$, as a function of sample temperature. While the peak fractional transmissivity change drops as $T_{c}$ is crossed, the change is not as dramatic as the relaxation time. In measured $d$-band metals, for photon energies close to the $d$-band-to-Fermi-level transition, the amplitude of the transmissivity change is directly related to the
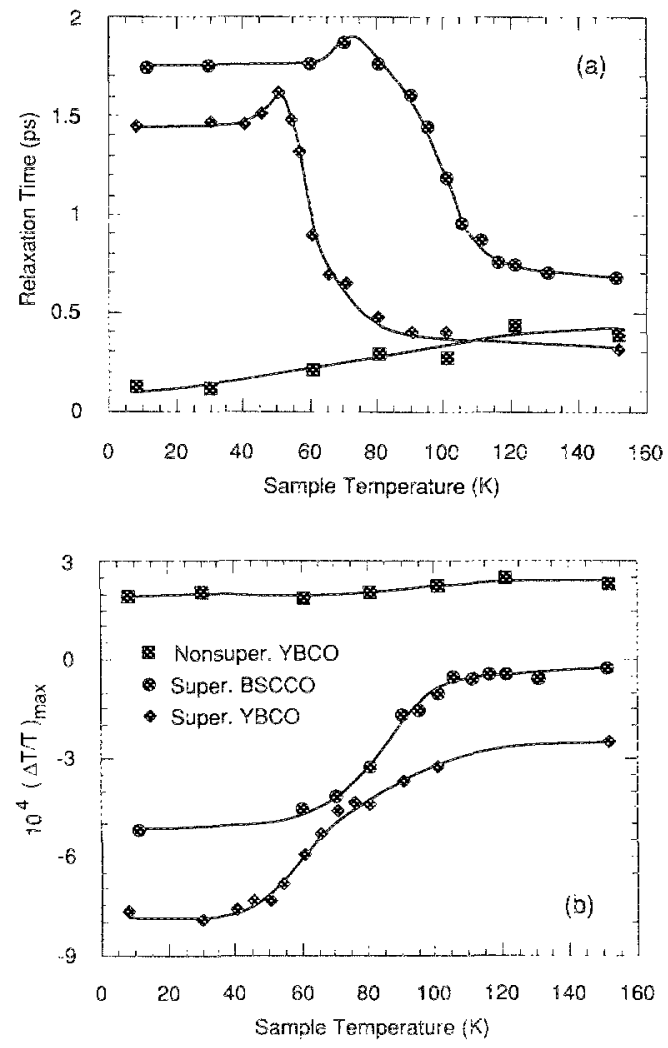

FIG. 2. (a) The relaxation tine as a function of sample temperature is shown for the superconducting BSCOO (\$), superconducting YBCO (), and nonsuperconducting YBCO (s) samples. (b) The peak fractional transmissivity change is plotted as a function of sample temperature for the films in (a). The peak fractional transmissivity change is negative for the BSCCO and superconducting YHCO samples, bat positive for the nonsuperconducting $\mathrm{YBCO}$ sample. In both figures the lines through the data points are meant only as a guide for the eye.

smearing of the electron distribution. The amount of smearing will change with sample temperature. However an abrupt change over a relatively small sample temperature range is not expected.' Also shown in Figs. 2(a) and 2(b) are data obtained from a superconducting $\mathrm{YBa}_{2} \mathrm{Cu}_{3} \mathrm{O}_{7-x}(x \sim 0)$ sample and a nonsuperconducting $\mathrm{YBa}_{2} \mathrm{Cu}_{3} \mathrm{O}_{6+,},(y<0.4)$ sample. Both YBCO samples came from the same thin-fim deposited on a MgO substrate produced using a standard laser ablation technique. The epitaxial thin film ( $\sim 0.2 \mu \mathrm{m}$ thickness) was $c$-axis oriented. An ac susceptibility measurement reveded an onset to diamagnetism at $87 \mathrm{~K}$. The oxygen content of the second sample was reduced rendering it nonsuperconducting by heating it at $420^{\circ} \mathrm{C}$ in flowing argon for $\sim 1 \mathrm{~h}$. Although the time domain data are not shown, similar behavior was observed with respect to the shapes and sample temperature in the superconducting YBCO sample, with the exception that the temperature dependence was shifted accordingly by the critical temperature.

Figure 3 plots the normalized transmissivity change for the nonsuperconducting YBCO sample at a few sample temperatures. The temporal response is quite different from the superconducting counterpart. This is not surprising since the nonsuperconducting phase is different structurally and electronically from the superconducting phase. The most distinguishing feature is the oscillation present in 


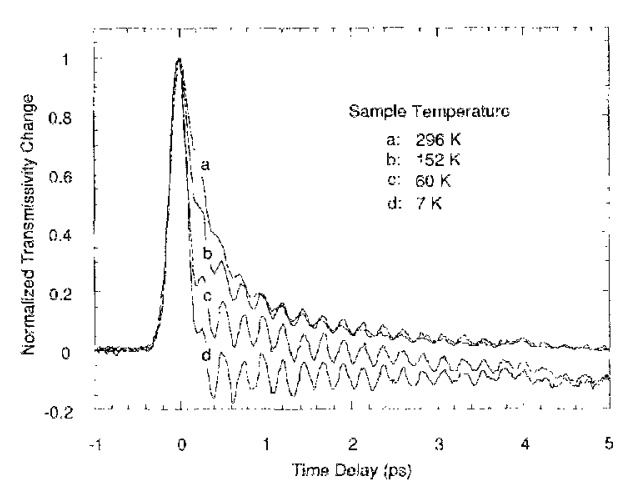

FIG. 3. Normalized transmissivity change for the nonsuperconducting YBCO sample is shown at a few sample temperarures. The zero delay time was chosen to coincide with the maximum transmissivity change of the $296 \mathrm{~K}$ trace.

the tail of the relaxation whose amplitude dampens with increasing time as well as with increasing sample temperature. The origin of these oscillations are not understood at this time. Interference effects seem unlikely since the round trip time of the light in the film $(\sim 4 \mathrm{fs})$ is much less than the laser pulse duration. We believe they may be an observation of coherent phonon oscillations. ${ }^{10}$ These ascillations are not observed in the superconducting compounds possibly because of increased scattering of the phonons with the much larger carrier concentrations. Another possibility is due to the structural difference; the associated mode may be present only in the nonsuperconducting compound. More studies are needed to confirm this. The other distinguishing feature that can be seen from Fig. 3 is the slight, only gradual temperature dependence in the relaxation. This is quantifed in Fig. 2(a), where the relaxation time is observed to increase slightly as the sample temperature increases. This trend continues to room temperature where the relaxation time of the nonsuperconducting sample is longer than its superconducting counterpart.

From Fig. 2(b) it can be seen that the peak fractional transmissiviey change is positive for the nonsuperconducting sample; this is opposite to the change measured for the superconducting samples. A reversal in the polarity of the transmissivity change in measured $d$-band metals is consistent with probing states in the vicinity of the Fermi level. However, the transmissivity change alone cannot determine the relative probing position with respect to the Fermi level. The important quantity for this determination is the change in the imaginary part of the dielectric function, $\Delta \epsilon_{2}$, which can be determined from simultaneous measurements of the fractional transmissivity and reflectivity changes. "This measurement was performed at room temperature. A positive $\Delta \epsilon_{2}$ consistent with probing unoccupied states below the Fermi level was found for the superconducting YBCO sample while a negative $\Delta \epsilon_{2}$ was found for the norsuperconducting YBCO sample. Due to the polycrystalline nature of the BSCCO film, obtaining accurate values of the optical parameters needed for determination of $\Delta \epsilon_{2}$ was difficult; most likely $\Delta \epsilon_{2}$ is positive. By removing the oxygen in the superconducting YBCO sample the number of carriers is reduced, which can depress the Fermi level. If the Fermi level is reduced sufficiently so that occupied states lying above the Fermi level are being probed, a polarity reversal will ensue. Although the Fermi surface for these materials is not well known, a sign reversal is consistent with probing states lying near the Fermi level, lending further support to the interpretation of the measured transients being a direct result of a nonequilibrium carrier distribution.

Because no structural phase transition is believed to accompany the entry into the superconducting domain in these high $T_{c}$ compounds and due to the extremely fast time scales involved, we believe the observed behavior is electronic in nature and intimately related to the superconductivity. If we are indeed probing states lying close to the Fermi level it is well known for BCS superconductors that as the material is cooled below its critical temperature the density of states exhibits an increase just below the Fermi level and a decrease at the Fermi level. This behavior corresponds to the opening of the energy gap in the superconductor. Evidence exists for this type of behavior occurring in the high $T_{c}$ superconductors through recent photoemission studies. ${ }^{2}$ This change in the density of states close to the Fermi level should then alter the Fermi smearing. ${ }^{13}$ More studies are needed to confirm this, in particular stucies to define the optical transition and correlate the measured transient change with the density of states at the Fermi level. It is possible to extend our studies to a broader wavelength range to actually map out, as a function of wavelength, the Fermi smearing as was done in Ref. 3 and to perform this both above and below $T$. This and related studies are currently under way.

This work was supported by the Air Force Office of Scientific Research, University Research Initiative under contract No. F49620-87-C-0016, NASA under contract No. NCC3-130, and the Eastman Kodak Company. We thank Hank Romanofsky for his technical assistance.

${ }^{1}$ G. L. Eeslcy, Phys. Rev. B 33, 2144 (1986).

${ }^{2}$ H. E. Elsayed-Ali, T. B. Norris, M. A. Pessot, and G. A. Mourou, Phys. Rev. Lett. 58, $1212(1987)$.

${ }^{3}$ R. W. Schoenlein, W. Z. I.in, J. G. Fujimoto, and G. L. Lesley, Phys. Rev. Iett. 58, 1680 (1987).

${ }^{4}$ G. L. Doll, G. L. Eesley, S. D. Brorson, M. S. Dresselhaus, G. Dresselhaus, A. Cassanho, H. P. Jenssen, and D. R. Gabbe, Appl. Phys. Lett. 55, 492 (1989).

'S. D. Erorson, A. Kazeroonian, D. W. Face, T. K. Cheng, G. L. Doll, M. S. Dresselhaus, G. Dresselhaus, E. P. Ippen, T. Venkatesan, X. D. Wu, and A. Inam, Solid State Commun. 74, 1305 (1990)

${ }^{6} \mathrm{D}$. van der Marel, J. van Elp, G. A. Sawatzky, and D. Heitmann, Phys. Rev. B 37, 5136 (1988)

${ }^{7}$ M. K. Kelly, P. Barboux, J.-M. Tarascon, and D. E. Aspnes, Phys. Rev. B 40, 6797 (1989).

${ }^{8}$ Estimated from the equations contained in A. Frenkel, M. A. Sait, $T$. Venkatesan, P. England, X. D. Wu, and A. Inam, J. Appl. Phys. 67, 3054 (1990).

${ }^{9}$ M. Leiental, S. Chen, S.-T. Lee, C. Braunstein, and P. Blanton, Physica C 167, 614 (1990).

${ }^{16}$ G. C. Cho, W. Kütt, and Ir. Kurtz, phys. Rev. Lett. 65, 764 (1990).

${ }^{11}$ R. Rosei and D. W. Lynch, Phys. Rev. B 5, 3883 (1972).

1)C. G. Olson, R. Liu, A.-R. Yang, D. W. Lynch, A. J. Arko, R. S. List, B. W. Veal, Y. C. Chang, P.-Z. Jiang, and A. P. Paulikas, Science 245, 731 (1989).

${ }^{13}$ R. Rosei, F. Antonangeli, and U. M. Grassano, Surf. Sci. 37, 689 (1973) 